\title{
Magia, humor y creatividad en el aula para potenciar el Pensamiento matemático
}

\author{
Ligia Stella Quemba Plazas*
}

Artículo de Investigación

Fecha de Recepción: 25 noviembre 2017

Fecha de Aprobación: 22 marzo 2018

\section{Resumen}

El proyecto de investigación Magia, humor y creatividad en el aula para potenciar el pensamiento matemático, es una estrategia didáctica que permite una profunda reflexión en el aula, para potencializar el pensamiento matemático en los estudiantes, empleando el humor y la magia como activadores de la creatividad, tomando la creatividad desde la relación del niño con el contexto. Cuando al estudiante se le estimula el pensamiento y la creatividad soluciona problemas matemáticos y problemas de situaciones de la vida diaria, pretende proponer modelos que aporten a brindar espacios propicios y significativos partiendo de una formación matemática no solo de tipo cognitivo, sino reflexivo, responsable, actualizado y coherente, que va más allá de los contenidos, desde una realidad contextualizada en miras que el estudiante desarrolle habilidades de imaginar, proponer y transformar, por ende tenga la capacidad de idear algo nuevo que permita resolver una situación matemática específica de manera innovadora y diferente capaz de analizar situaciones de la vida cotidiana, sacando conclusiones propias sobre las mismas que les permita, evaluar y cuestionar lo que nos rodea, con el fin de actuar de manera más inteligente.

* Institución donde labora Boyacá - Colombia ligiaquemba@hotmail.es

Palabras claves: pensamiento matemático, creatividad, humor y magia.

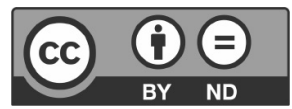




\section{Introducción}

Para formar estudiantes en ciudadanos matemáticamente competentes, este debe tener dominio de los procesos, competencias y procedimientos que se requieren en los cinco tipos de pensamiento matemático los cuales van coherentemente relacionados entre sí. Afirma LLinares (2010) "el pensamiento matemático de los estudiantes implica identificar los aspectos relevantes e interpretarlos para dotarlos de significado y poder tomar decisiones de acción”, según Molina (2006) "pensamiento matemático es la actividad intelectual mediante la cual el hombre entiende, comprende y dota de significado lo que lo rodea". Desarrollar el pensamiento matemático es un reto para el ministerio de educación nacional, es una problemática que se presenta a nivel nacional, departamental y municipal como es el caso del municipio de Gameza en la Institución Educativa Juan José Reyes Patria, es una institución de carácter oficial, basada en el modelo pedagógico constructivista con enfoque por competencias ofrece los grados de preescolar, básica y media, institución ofrece dos Programas de Articulación con la Educación Superior, con el SENA en Seguridad Ocupacional y Técnico en Sistemas, educación de jóvenes y adultos.

La población objeto de estudio son originarios $100 \%$ del municipio, y residen entre el sector urbano y rural, los padres de familia se caracterizan por su nivel socioeconómico entre los estratos 1 y 2 su economía gira en torno a la producción agrícola y minería de carbón no tecnificada, nivel de escolaridad no es completo. La Institución Educativa cuenta con siete sedes San Laureano donde funciona la básica y media sedes rurales: Guanto, San Antonio, Potosí, Motua, Satoba, Villa Girón, y la sede urbana de primaria Francisco José de caldas, Para la investigación se tomó una Población y muestra, de 21 estudiantes de grado $3 \mathrm{~B}$, de la sede caldas, el diagnóstico realizado en el contexto escolar, el bajo rendimiento en: el área de matemáticas, resultados de las pruebas saber, el índice sintético de educación nacional y pruebas internas, Los estudiantes no tiene dominio de los estándares básicos de competencias en el área de matemáticas, no hay una comprensión e interpretación de proceso de los cinco tipos de pensamiento matemático, en el análisis de las pruebas saber, se obtuvo los siguientes resultados 2016 (4.97) y un progreso de 00 , se puede observar (tabla 1) del Índice Sintético de Calidad Educativa, datos tomados del Ministerio de Educación Nacional. En esta Institución Educativa se evidencia que en el Proyecto Educativo Institucional, no hay una secuencia del modelo pedagógico y de metodología, en primaria se trabaja con escuela nueva en las rurales y en la urbana escuela graduada, Los padres de familia afirman por medio de charlas que: no hay concientización por parte del docente a que los estudiantes sean responsables en la realización de sus labores escolares, El bajo rendimiento académico es el factor que conlleva a la desmotivación del estudiante, Los docentes mediante charlas afirman: que los estudiantes 
no tienen responsabilidad para realizar tareas y trabajos extra clase, los estudiantes de primaria vienen mal preparados en las áreas de matemáticas y por ende hay deserción en sexto. Toda esta problemática se encierra en la pregunta generalizadora donde se centra la investigación ¿Cómo desarrollar el pensamiento matemático a través de una propuesta didáctica mediada por la creatividad en los niños del grado tercero de la Institución Educativa Juan José Reyes Patria del municipio Gameza?

Se ve la necesidad de implementar acciones y estrategias didácticas para retomar los aprendizajes en el área de matemáticas, que va más allá de los contenidos desde una realidad contextualizada. Por ende, Se viene desarrollando el proyecto pensamiento matemático en básica primaria, el estado del arte se hizo por triangulación de datos, tomando aportes de: Jean Piaget, Sigmund Freud, Taylor, Edwar de Bono, Cantoral, Molina, LLinares, Gardner, Csikszentmihalyi, entre otros. Buscando dar respuesta al interrogante de investigación, por tanto, el objetivo general Desarrollar el pensamiento matemático a través de una propuesta didáctica mediada por la creatividad en los niños del grado tercero de institución educativa Juan José Reyes Patria del municipio de Gameza en el departamento de Boyacá. Tomando la creatividad desde la relación del niño con el contexto, Cuando al estudiante se le estimula el pensamiento y la creatividad va ser competente en crear y solucionar problemas matemáticos y problemas de situaciones de la vida diaria, afirma Csikszentmihalyi (1988) "La creatividad no se produce dentro de la cabeza de las personas, sino en la interacción entre los pensamientos de una persona y un contexto sociocultural”. Según Arias (2002) donde tiene en cuenta la creatividad en los procesos matemáticos, "el matemático frente a un problema, ensaya, yerra, vuelve a atacar con otro esquema y en general necesita una gran imaginación y creatividad". La Metodología empleada: enfoque cualitativo, tipo de investigación acción, tomada desde el modelo Elliott (1981), Paradigma crítico social, se emplea instrumentos como: la Observación directa, Charlas con docentes donde relatan sus prácticas, cuestionarios, aplicación de pruebas diagnósticas, y de rendimiento, colaboración de los doctores salvador LLinares y Ricardo Cantoral mediante correo electrónico, han brindado grandes aportes a este proyecto. Para llevar registro, y recopilación de información en: audio, video, y diario de campo. La estrategia didáctica se elaboró teniendo en cuenta el contexto educativo y el diagnóstico de la problemática identificada, Se diseñó actividades que se ejecutarán en las fases de socialización, intervención, seguimiento, En cada una de estas fases se desarrollan acciones estratégicas coordinadas, que permitan: el conocimiento, la reflexión, e interacción, se evaluará el proceso didáctico, antes, durante, y después. Se realizó consolidación del diagnóstico claro y preciso de la problemática de la institución Educativa, elaboración de una estrategia didáctica mediada por la creatividad, e inicio de la
En el nivel literal, se encontró que, al principio, 17 de los 33 estudiantes tenían una valoración baja; y 4, una valoración alta. 
Implementación de la estrategia, con el fin de lograr el objetivo planteado. En la estrategia para activar la creatividad se empleará el humor y la magia Conde (2010) "La Magia como instrumento pedagógico posee un gran valor intelectual. Enseñando
Magia a los niños desarrollamos su sentido de la observación y valoración. Disciplinamos su mente y cuerpo, potenciamos la creatividad e imaginación y resulta una herramienta excelente para vencer la timidez".

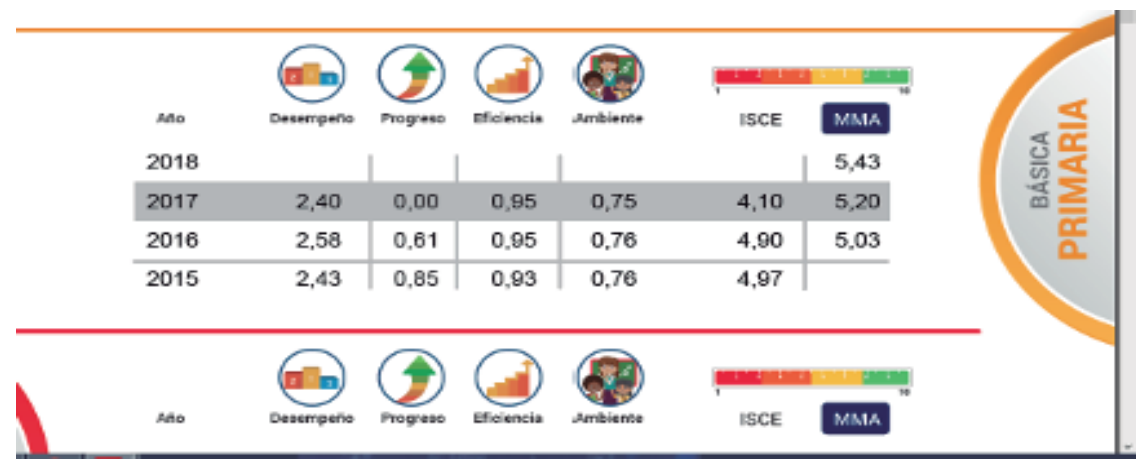

Tabla 1 Índice Sintético de Calidad Educativa

\section{Objetivo general}

Desarrollar el pensamiento matemático a través de una propuesta didáctica mediada por la creatividad en los niños del grado tercero de institución educativa Juan José Reyes Patria del municipio de Gameza en el departamento de Boyacá.

\section{Objetivos específicos}

Realizar un diagnóstico claro y seguro caracterizando en el ISCE, la problemática del bajo rendimiento de las pruebas saber en la I.E Juan José Reyes Patria.

Elaborar una propuesta didáctica teniendo en cuenta el contexto educativo y el diagnóstico de la problemática identificada.
Implementar una propuesta didáctica para mejorar el pensamiento matemático mediado por la creatividad.

Evaluar el proceso didáctico mediado por la creatividad antes, durante, $y$ después.

\section{Referentes teóricos}

\section{Pensamiento Matemático}

Para el desarrollo del proyecto de investigación, se tienen en cuanta los referentes teóricos iniciando con la definición de pensamiento matemático, para Piaget, "pensamiento operatorio concreto "afirma que el pensamiento lógico actúa por medio de operaciones y el pensamiento matemático sobre el número y el espacio. Para Cantoral (2005) pensamiento matemático, parte 
de un ámbito científico en el cual los conceptos y las técnicas matemáticas surgen y se desarrollan en la resolución de tareas. Molina (2006) afirma que el pensamiento matemático es la actividad intelectual mediante la cual el hombre entiende, comprende y dota de significado lo que lo rodea. LLinares, (2010) "el pensamiento matemático de los estudiantes implica identificar los aspectos relevantes e interpretarlos para dotarlos de significado y poder tomar decisiones de acción”, Pérez y Gardey. (2011) definen el pensamiento matemático en la sistematización y la contextualización del conocimiento de las matemáticas, se desarrolla a partir de conocer el origen y la evolución de los conceptos y las herramientas que pertenecen al ámbito matemático. El pensamiento matemático, por lo tanto, incluye conocer cómo se ha ido formando un concepto o técnica. De esta manera, la persona conoce sus dificultades inherentes y descubre como explotar su uso de forma adecuada.

Desde el Ministerio de Educación Nacional MEN, Estándares básicos de competencias selecciona avances en el desarrollo de las competencias asociados con los cinco tipos de pensamiento matemático (numérico, espacial, métrico, aleatorio y variacional). Derechos Básicos de Aprendizaje v2. La educación de calidad es un derecho fundamental y social que debe ser garantizado para todos. Presupone el desarrollo de conocimientos, habilidades, y valores que conforman a la persona de manera integral.
El proyecto secentra en La problemática que hay en la formación matemática, es una realidad social donde se debe centrar en las prácticas docentes y en el aprendizaje de los estudiantes. Los estudios realizados sobre el pensamiento matemático se tienen en cuenta en el proyecto caracterizado por estudios internacionales, latinoamericanos y nacionales.

\section{Internacional}

A mediados del siglo xx Jeans Piaget realizo estudios de la manera de razonar de los adolescentes, Piaget (1985) en los estudios realizados por Piaget sobre la lógica y la epistemología propone que "el pensamiento lógico actúa por medio de operaciones sobre las proposiciones y el pensamiento matemático se distingue del lógico porque versa sobre el número y sobre el espacio". Investigaciones se centran en los estudiantes y maestro interpretan comprenden los contenidos matemáticos estudios realizados por LLinares et al (2010) "La competencia docente del maestro "mirar con sentido" el pensamiento matemático del estudiante implica identificar los aspectos relevantes e interpretarlos para dotarlos de significado y poder tomar decisiones de acción”.

\section{Latino americana}

Se toma como referente al doctor mexicano Cantoral (2002), donde recalca la importancia de la didáctica del docente, "En el sistema escolar la preocupación está basada en la enseñanza y a su medida, se diseñan
El proyecto se centra en $\mathrm{La}$ problemática que hay en la formación matemática, es una realidad social donde se debe centrar en las prácticas docentes y en el aprendizaje de los estudiantes. 
actividades escolares sin entender a los factores del aprendizaje en matemáticas".

\section{Nacional}

Los aportes Luque (2002) donde este autor tiene en cuenta la creatividad en los procesos matemáticos, "el matemático frente a un problema, ensaya, yerra, vuelve a atacar con otro esquema y en general necesita una gran imaginación y creatividad".

Ministerio de Educación Nacional en el foro nacional de matemáticas (2014) Es una realidad que la imagen social hacia las matemáticas, los matemáticos y los docentes de matemáticas es muy negativa. Para un amplio sector de la sociedad las matemáticas son difíciles, inútiles y poco comprensibles. A diario los estudiantes toman decisiones sobre su vida futura teniendo como referente la fobia hacia las matemáticas.

En el 2006 el Ministerio de Educación Nacional en el documento No3 cita los

Cinco tipos de pensamiento matemático Ministerio de educación Nacional en los lineamientos curriculares de matemáticas propone, "el saber hacer en contexto" el ser competente en matemáticas requiere de eficacia y eficiencia el pensamiento matemático, el cual se subdivide en los cinco tipos de pensamiento el numérico, el espacial, el métrico o de medida, el aleatorio o probabilístico y el variacional, los cuales van relacionados y articulados entre sí, tiene elementos conceptuales comunes. (Ministerio de Educación Nacional, 2006, p.56)

\section{Pensamiento numérico y sistema numérico}

Para el desarrollo de este pensamiento el ministerio de educación nacional en los lineamientos curriculares, las actividades se deben centrar en la comprensión, uso y significado de los números, las operaciones y sus relaciones con los números, y el desarrollo de los diferentes técnicos de cálculo y estimación, el empleo de las operaciones adición sustracción, multiplicación y división. Con el acompañamiento pedagógico y progresivo los estudiantes pueden lograr la construcción y progreso del conocimiento y dominio del pensamiento numérico. (Ministerio de Educación Nacional,2006, p 58)

\section{Pensamiento espacial y los sistemas geométricos}

El pensamiento espacial es el conjunto de los procesos cognitivos mediante los cuales se construye las representaciones mentales de los objetos del espacio, es importante las relaciones entre los objetos en el espacio y la ubicación y relación del individuo con respecto a estos.

El estudio de la geometría se relaciona con el arte, decoración, el diseño y construcción de objetos artesanales y tecnológicos educación física los deportes las danzas, el trabajo con objetos bidimensionales tridimensionales, la geometría es una herramienta de exploración y de representación del espacio. ((Ministerio de Educación Nacional, 2006, p.61) 
Pensamiento métrico y los sistemas métricos y de medidas

Hace referencia a la comprensión de magnitudes cantidades medición y uso flexible delos sistemas métricos o de medidas, el pensamiento métrico está relacionado con las disciplinas científicas naturales y sociales competencias ciudadanas, cuidado del medio ambiente, dar especial importancia a estas magnitudes que tiene estrecha relación con aspectos de la vida social del estudiante. (Ministerio de Educación Nacional, 2006, p63).

Pensamiento aleatorio y los sistemas de datos

Este pensamiento es llamado también pensamiento probabilístico o escolástico, pensamiento se apoya directamente en conceptos y procedimientos de la teoría de probabilidades y de la estadística inferencial ayuda a buscar soluciones razonables a problemas en los que no hay una solución clara y segura, el azar se relaciona con la ausencia de patrones o esquemas específicos en las repeticiones de eventos o sucesos. (Ministerio de Educación Nacional, 2006, p63).

\section{Pensamiento variacional y los sistemas algebraicos y analíticos}

Este pensamiento tiene que ver con el reconocimiento, la percepción la identificación y la caracterización de la variación y el cambio en diferentes contextos, con la descripción, modelación, y representación en distintos sistemas o registros simbólicos, ya sean verbales, iconos, gráficos, o algebraicos, el estudiante debe comprender el uso de los conceptos y procedimientos de las funciones y sus temas analíticos para el aprendizaje del cálculo numérico y algebraico. Para el desarrollo de este pensamiento es importante presentarle actividades de análisis de cambios, aumenta disminuye la forma o el valor de una secuencia o sucesión de figuras, números o letras (página 63).

\section{El Ministerio de Educación Nacional en el documento No 3 cita los Procesos generales de actividad matemática}

En los lineamientos curriculares de área de matemáticas los procesos son cinco: formular y resolver problemas; modelar procesos y fenómenos de la realidad; comunicar; razonar; formular comparar y ejercitar procedimientos y algoritmos.

Pensamiento matemático y la creatividad

La creatividad es una habilidad que se puede aprender, desarrollar y aplicar, hace la vida más divertida por lo que no hay repetición ni rutina, se puede desarrollar con trabajo constante y con disciplina y además estar muy motivado, para llegar a ser creativo se necesitan operaciones mentales contenidos mentales y productos mentales donde el individuo codifica, selecciona combina y compara es lo que se necesita que desarrolle el niño para su aprendizaje, en las diferentes áreas del conocimiento acompañada de la imaginación de imaginar situaciones cosas nueva invente cosas que no existen o existían el niño tiene que tener un acompañamiento una motivación por parte del maestro el niño va desarrollar competencias y habilidades donde sienta la necesidad de cambio, que se ha creativo y critico 
Freud (1963): "La creatividad se origina en un conflicto inconsciente. La energía creativa es vista como una derivación de la sexualidad infantil sublimada, y que la expresión creativa resulta de la reducción de la tensión"

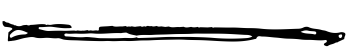

a su entorno y cree nuevas cosas genere ideas El niño tiene que ser motivado y el contexto es importante para que desarrolle esta capacidad.

Al realizarle al estudiante actividades orientadas, Son muchas las técnicas que se han realizado para estimular la producción de ideas y habilidades creativas las cuales son de gran importancia se lleve al aula de clase. Se necesita personas creativas que den soluciones ideas nuevas pero que estas llevan un orden una sistematización.

Desarrolla habilidades comunicativas donde se va a reflejar en su oralidad la crítica constructiva. Aprender a su propio ritmo y la manera que más le guste donde el docente este para ayudar a dar soluciones a los problemas que no pueda resolver y sea un orientador formador de ideas, donde esas ideas, sueños de los niños se hagan realidad. El aula debe permitir condiciones favorables para estimular la creatividad de los niños, es donde se debe desarrollar esa potencialidad, El proyecto Toma la creatividad desde la relación del niño con el contexto, afirma Csikszentmihalyi. (1988) "La creatividad no se produce dentro de la cabeza de las personas, sino en la interacción entre los pensamientos de una persona $y$ un contexto sociocultural". Según Luque Arias (2002) donde tiene en cuenta la creatividad en los procesos matemáticos, "el matemático frente a un problema, ensaya, yerra, vuelve a atacar con otro esquema y en general necesita una gran imaginación y creatividad". Ausubel (1963): "La personalidad creadora es aquella que distingue a un individuo por la calidad y originalidad fuera de lo común de sus aportaciones a la ciencia, al arte, a la política, etcétera" (Psicólogo y pedagogo. Destaca la novedad y el valor. Es uno de los máximos representantes del constructivismo).

Freud (1963): "La creatividad se origina en un conflicto inconsciente. La energía creativa es vista como una derivación de la sexualidad infantil sublimada, y que la expresión creativa resulta de la reducción de la tensión”, Piaget (1964): "La creatividad constituye la forma final del juego simbólico de los niños, cuando éste es asimilado en su pensamiento" (Epistemólogo, psicólogo y biólogo. Reconocido por sus teorías sobre el desarrollo cognitivo y la inteligencia). Taylor (1971): "La Creatividad, implica una variedad de procesos y percepciones dirigidas a alterar y reorganizar una porción significativa del ambiente en concordancia con los patrones de la propia persona o la estructura de necesidades, hipótesis, juicios y percepciones, proporcionando una alteración que es única o no común y relevante al problema." Y en 1975: "El proceso intelectual que tiene por resultado la producción de ideas, a la vez nuevas y valiosas" (Integra novedad y valor).De Bono, (1992) "El pensamiento creativo. El poder del pensamiento lateral para la creación de nuevas ideas".

El humor y las matemáticas

La estrategia se trabajará el humor, como un activador de la creatividad, 
Pelayo () dice: "el chiste desarrolla el sentido del humor del niño, con el sentido del humor desarrollado, el niño vivirá con un estado de ánimo positivo con buen humor con disposición y viceversa, porque es un circulo virtuoso. Con este talante positivo el niño estará más dispuesto a entregarse a cualquier actividad placentera, lúdica. Con el sentido del humor desarrollado, también el niño desarrollara su sentido crítico su sentido común, su imaginación, su creatividad y mejorara su personalidad". El aula debe permitir condiciones favorables para estimular la creatividad de los niños, es donde se debe desarrollar esas potencialidades para que haya un dominio y manejo de cinco tipos de pensamiento matemático.

Magia y matemáticas

Conde (2016). "La Educación es Magia. La Magia es ilusión y la ilusión es el verdadero motor de la vida" Con la magia se proponen situaciones matemáticas para que el estudiante descubra su procedimiento y posibles soluciones mediante la investigación, observación y experimentación e Identifiquen las características didácticas de la magia como un medio para propiciar el aprendizaje del pensamiento numérico, métrico y espacial. El docente realizara trucos, la magia es muy útil para que los estudiantes estén atentos y curiosos buscan la manera de descubrir el truco, empieza a investigar ¿de dónde aparecen y desaparecen cosas? ¿Por qué cambian? En la mente del niño se hace cuestionamientos y empieza a investigar se amplía el pensamiento crítico para llegar al proceso de producción, planteando situaciones problema. Conde (2016) "la magia educativa también desarrolla el pensamiento lógico matemático $\mathrm{El}$ docente debe tener cualidades de creativo, ofrecer un ambiente innovador e inolvidable que cada aprendizaje sea un momento mágico para el estudiante”, Suarez (2010) "el acto de magia debe ser pensado con base en su público; en especial, cuando de niños se trata hay que preparar juegos fantásticos, con historias, implementos y efectos llamativos y avasalladores, para nunca olvidar". Conde (2014) "La magia a través de estas palabras, consigue abrir la mente de niños y educadores hacia el conocimiento y la educación. La magia resulta un elemento motivador y divertidísimo para los niños y adultos y esto les permite desarrollar Sus capacidades, habilidades y competencias sin apenas darse cuenta y de forma totalmente lúdica”.

\section{Metodología}

La investigación tiene enfoque cualitativo, método inductivo, Paradigma critico social. Referenciando a Paulo Freire apunta a ligar el proceso de aprendizaje con el uso social real del conocimiento como herramienta (empoderamiento). La pedagogía crítica busca enfrentar a estudiantes y docentes a los problemas de la realidad objetiva en la que vive y se desarrolla; por lo que la fuente fundamental para la selección de los contenidos es la propia realidad social en estrecho vínculo con la comunidad donde se encuentra 
ubicada la escuela. Freire (1979) "La acción crítica es la praxis, que se mueve entre la reflexión y la acción”, los instrumentos para la recolección de la información son: Observación directa; tipo Investigación acción tomada desde el modelo de John Elliott, Observación directa contacto directo con el objeto de estudio, la recopilación de información y la recolección de las evidencias en el diario de campo de la docente portafolio y cuaderno de los estudiantes, donde se sistematizara lo observado y las actividades realizadas, análisis del Índice Sintético de Calidad Educativa, pruebas de rendimiento supérate con el saber, pruebas saber de los últimos años, pruebas internas de Milton Ochoa martes de prueba, material recibido del doctor Salvador LLinares (comunicación personal, 30 de Mayo /2017) este material para adaptarlo a prueba diagnóstica. Población y muestra: estudiantes del grado tercero de primaria de la Institución Educativa Juan José Reyes Patria del municipio de Gameza, tomándose una muestra de 21 niños los cuales representa el 100\% de los estudiantes de grado $3 \mathrm{~B}$ de la sede Francisco José de Caldas, el 50\% del total de estudiantes del grado tercero, de esta sede.

A continuación, se describe las fases de la investigación.

La investigación se desarrolla en tres fases con el propósito de lograr el objetivo propuesto:

Fase diagnostica se emplearon instrumentos de análisis para obtener un diagnóstico claro y seguro de la problemática, caracterizando en el
ISCE, se aplicaron varios instrumentos para validar el diagnóstico, donde surge la pregunta de investigación. Para el estado del arte se realizó investigación de diferentes autores, por triangulación de datos se eligieron los autores antes mencionados, se archiva en el portafolio Los consentimientos firmados por los padres autorizando la participación de los niños en el proyecto.

Fase de elaboración e implementación de una propuesta didáctica mediada por la creatividad Se elaboró una propuesta didáctica mediada por la creatividad teniendo en cuenta el contexto educativo y el diagnóstico de la problemática identificada, los saberes previos, las potencialidades y actitudes de los estudiantes.

Fase de evaluación Para evaluar los procesos de la investigación se realizará entre fases antes durante y después, teniendo en cuenta la autoevaluación coevaluación, estas intervenciones ayudaran a determinar los aportes generados por cada uno de los instrumentos aplicados para ir realizando planes de mejoramiento.

\section{Resultados y discusiones}

Los resultados de la investigación son preliminares, es un proyecto de aula que continua en proceso de elaboración, se realizó diagnóstico claro y seguro caracterizando en el Índice sintético de calidad Educativa, sus respectivos componentes y la meta de mejoramiento a alcanzar se aplicaron las pruebas supérate con el saber del año 2017, las pruebas saber 
de los años 2014,2015,2016 martes de pruebas de Milton Ochoa, cuadernillo de actividades proyecto sé, pruebas con adaptaciones del material del Doctor Salvador LLinares, donde se pudo diagnosticar que los estudiantes: no construyen ni describen secuencias numéricas y geométricas, no clasifican ni organizan la presentación de datos, no ubican objetos con base en instrucciones referentes a dirección, distancia y posición, no establecen correspondencia entre objetos o eventos o patrones $\mathrm{o}$ instrumentos de medida, no usan operaciones ni propiedades de los números naturales para establecer relación entre ellos en situaciones específicas, no resuelven ni formulan situaciones multiplicativas. Una de las fortalezas en algunos de los estudiantes sabe mecánicamente sumar y restar, pero no saben los procesos y su utilidad en situaciones problema que se les presenta, los resultados de informes bajo rendimiento académico en el área de matemáticas.

Se elaboró una propuesta didáctica mediada por la creatividad teniendo en cuenta el contexto educativo y el diagnóstico de la problemática identificada, y los saberes previos, las potencialidades y actitudes de los niños. formando niños con ideas nuevas, con la habilidad de pensar crítica y creativamente desarrollando el pensamiento matemático para que sea competentemente matemático, ofrecer un ambiente donde el estudiante experimente un aprendizaje significativo y descubra su propio aprendizaje, en la fase dos Se socializó el proyecto a docentes y directivos hay disponibilidad a colaborar y dar aportes de sus experiencias al proyecto, se adecuo el aula creando una aula didáctica con material elaborado por los niños y la docente, donde podemos encontrar artefactos como: el yapay y multilego, instrumentos de madera para facilitar el aprendizaje de las operaciones básicas. en el rincón de las matemáticas se ubicó material didáctico: dados, fichas, cubos, regletas de colores, geoplanos, reglas marcadoras entre otros.

La posible limitación falta de colaboración por parte de algunos padres de familia para el desarrollo de actividades propuestas, la no apropiación y participación del proyecto por parte de algunos los docentes de básica,

\section{Conclusiones}

Al implementar la estrategia mediada por la creatividad se espera que se creen ambientes propicios de enseñanza y aprendizaje para potenciar desarrollo del pensamiento matemático, con el propósito de aportar a la educación y al verdadero aprendizaje de las matemáticas formando niños con ideas nuevas, con la habilidad de pensar crítica y creativamente desarrollando el pensamiento matemático para que sea un ciudadano matemática mente competente, al adecuar el aula y los estudiantes aportan para que se cree un ambiente donde se experimente un aprendizaje significativo y descubran su propio aprendizaje, a los estudiantes se les dificulta el trabajo colaborativo y cooperativo al adaptar el aula se
Se elaboró una propuesta didáctica mediada por la creatividad teniendo en cuenta el contexto educativo y el diagnóstico de la problemática identificada, y los saberes previos, las potencialidades y actitudes de los niños 
hizo cambio de pupitres unipersonales por mesas hexagonales difícil su adaptación en el trabajo en equipo se presentó mucho indisciplina al iniciar con la estrategia, el estudiante venía con un modelo tradicional de trabajo individual, se designó un monitor para darle responsabilidades y liderazgo el cual se rota diariamente, formación de estudiantes con habilidades, actitudes, valores, la estrategia ofrece herramientas necesarias para enfrentar las pruebas internas y externas para alcanzar mejores resultados.

\section{Referentes}

Piaget, J. (1973). La formación del símbolo en el niño. Fondo de cultura económica.

De Bono, E. (1986). El pensamiento lateral. Editorial Paidós, Barcelona

De Guzmán, M. (1992). Tendencias innovadoras en educación matemática. Olimpíada Matemática Argentina.

Ministerio de Educación Nacional (1988). Matemáticas Lineamientos curriculares.

De Guzmán Ozámiz, M. (2006). Para pensar mejor: desarrollo de la creatividad a través de los procesos matemáticos.

Suarez, M. (2010). La magia y una nueva relación pedagógica con la infancia. Praxis \& Saber, 1(2), 43-66. https://doi.org/10.19053/22160159.1098

Llinares S (2011). El desarrollo de un esquema para caracterizar la competencia docente" mirar con sentido" el pensamiento matemático de los estudiantes. In Investigación en educación matemática XV (pp. 351-360).

Llinares, A. Z., \& de la Vega, M. L. C. (2013). Cómo interpretan los estudiantes para maestro el pensamiento matemático de los alumnos sobre el proceso de generalización. In Investigación en Educación Matemática XVII (pp. 535544). Servicio de Publicaciones.

Malaspina, U. (2013). La enseñanza de las matemáticas y el estímulo a la creatividad. UNO, Revista de Didáctica de las Matemáticas, 63, 41-49.

Ministerio de educación Nacional (2014) memorias del foro Nacional de matemáticas, matemáticas para todos y con todos. 